\title{
Role of Hydroxyapatite and Ellagic Acid in the Osteogenesis
}

\author{
Agung Satria Wardhana ${ }^{2}$ Intan Nirwana ${ }^{2, \odot}$ Hendrik Setia Budi ${ }^{3, \odot ~ M e i r c u r i u s ~ D w i ~ C o n d r o ~ S u r b o y o ~}{ }^{4, \odot}$
}

\author{
${ }^{1}$ Department of Dental Material, Faculty of Dentistry, \\ Universitas Lambung Mangkurat, Banjarmasin, Indonesia \\ ${ }^{2}$ Department of Dental Material, Faculty of Dental Medicine, \\ Universitas Airlangga, Surabaya, Indonesia \\ ${ }^{3}$ Department of Oral Biology, Faculty of Dental Medicine, \\ Universitas Airlangga, Surabaya, Indonesia \\ ${ }^{4}$ Department of Oral Medicine, Faculty of Dental Medicine, \\ Universitas Airlangga, Surabaya, Indonesia
}

\author{
Address for correspondence Intan Nirwana, drg., M.Kes, \\ Department of Dental Material, Faculty of Dental Medicine, \\ Universitas Airlangga, Surabaya 60132, Indonesia \\ (email: Intan-n@fkg.unair.ac.id).
}

Eur J Dent:2021;5:8-12

\author{
Abstract \\ Keywords \\ - osteogenesis \\ - hydroxyapatite \\ - ellagic acid \\ - osteocalcin \\ - RANKL \\ $-O P G$
}

Objective Ellagic acid (EA), a phenolic antioxidant, has benefits in bone health and wound healing. The combination of EA and hydroxyapatite (HA) (EA-HA) is expected to increase osteogenesis. The aim of this study was to analyze osteogenesis after application of EA-HA according to the number of osteoblasts and osteoclasts in the bone and the expression of the receptor activator of nuclear factor kappa-B ligand (RANKL), osteoprotegerin (OPG), and osteocalcin (OCN) protein.

Materials and Methods Thirty Wistar rats were assessed with bone defects created in the left femur. The defects were filled with EA-HA and then sutured. Control groups were filled with polyethylene glycol (PEG) or HA. Each group was sacrificed either 7 or 14 days after treatment.

Results The defects filled with EA-HA exhibited the highest number of osteoblasts and the greatest expression of OPG and OCN at both day 7 and day $14(p=0.000)$. Conversely, treatment with EA-HA resulted in lower numbers of osteoclasts and reduced RANKL staining at both time points $(p=0.000)$.

Conclusions EA-HA can increase osteogenesis in bone defects by increasing the number of osteoblasts and the expression of OPG and OCN.

\section{Introduction}

As periodontal diseases progress, excess bone resorption can result in bone defects. Periodontal bone defects are highly prevalent in Indonesia, where the incidence has increased throughout the last decade. ${ }^{1,2}$ According to data from Riset Kesehatan Dasar, the prevalence of this disease in 2007 was 23.4\%. This increased to $25.9 \%$ in 2013 and the most recent data in 2018 reports a prevalence of $57.6 \%$. This is similar to the United States, where $50 \%$ of the adult population suffer bone resorption as periodontal diseases progress. The Indonesian Ministry of Health Survey in 2011 stated that bone defects and periodontal disease account for $60 \%$ of oral health problems in Indonesia.

The progression of bone defects into the alveolar bone can necessitate the wearing of complete dentures, especially if the defect is in the mandibular region of the anterior region, which is four times more common than in the alveolar bone of the maxilla. ${ }^{3}$ The most commonly used therapy, especially in cases of large bone defect, is to use a bone graft. This graft can be either an autograft, an allograft, or a xenograft. ${ }^{4}$ The most common source for a xenograft, which is a graft taken from another species, is bovine bone, which is then processed until pure hydroxyapatite (HA) mineral is obtained.
DOI https://doi.org/ 10.1055/s-0040-1714039 ISSN 1305-7456.
(C) 2020. European Journal of Dentistry.

This is an open access article published by Thieme under the terms of the Creative Commons Attribution-NonDerivative-NonCommercial-License, permitting copying and reproduction so long as the original work is given appropriate credit. Contents may not be used for commercial purposes, or adapted, remixed, transformed or built upon. (https://creativecommons.org/licenses/by-nc-nd/4.0/)

Thieme Medical and Scientific Publishers Pvt. Ltd., A-12, 2nd Floor, Sector 2, Noida-201301 UP, India 
This material is a good osteoconductor, with ratios of calcium and phosphate that are suitable for use in humans. ${ }^{5-7} \mathrm{HA}$ is one of the materials commonly used for bone grafts and is easily obtained from bones, teeth, or natural minerals. HA is an inorganic compound found in human bones and teeth. In fact, almost $95 \%$ of tooth enamel is HA in the form of calcium HA crystals. ${ }^{8}$ Therefore, HA can be used in the form of a graft or inert scaffold for bone healing because its chemical structure resembles natural bone. In addition, HA has good biocompatibility, bioaffinity, bioactivity, osteoinduction, osteoconduction, and osteointegration properties. ${ }^{5,9}$

Bone damage triggers the process of bone healing that begins with inflammation. This inflammation increases the activity of the osteoclasts that play a role in bone resorption. Suppressing inflammation would be expected to decrease osteoclast activity and so increase the rate of new bone formation..$^{10}$ The inclusion of factors such as ellagic acid (EA) can be considered to add anti-inflammatory properties to bone graft material. EA is a compound that is commonly found in pomegranates alongside other polyphenol compounds such as Gallo tannin and anthocyanin. ${ }^{11}$ Polyphenol compounds are the most important bioactive materials in the maintenance of bone health. ${ }^{12}$ EA prevents the formation of free radicals and has anti-inflammatory, antioxidant, antiapoptotic, antimutagenic, and antiviral properties. ${ }^{10}$

There are no reports into the effects of the combination of EA and HA on the bone healing process. Therefore, it is necessary to study whether the combination of EA and HA affects the process of osteogenesis associated with bone defects. Effects on osteogenesis can be observed via the expression of bone growth biomarkers such as receptor activator of nuclear factor kappa-B ligand (RANKL), which binds to the cytokine osteoprotegerin (OPG) that is produced by osteoblasts and acts as a decoy receptor for RANKL. Osteocalcin $(\mathrm{OCN})$ is another important component of bone formation. This study will generate data that will be useful for the development of future research in the fields of biomaterials and tissue regeneration.

\section{Materials and Methods}

\section{Ethical Clearance}

This research protocol was approved by the Ethics Committee of the Faculty of Dental Medicine at the University of Airlangga (431/HRECC.FODM/VII/2019).

\section{Materials and Antibodies}

HA powder (BATAN, Jakarta, Indonesia); EA (90\%, Xi'an Biof Bio-Technology, Shaanxi, China); polyethylene glycol (PEG, 202398, Sigma-Aldrich); anti-RANKL polyclonal antibody (ab216484, Abcam); anti-OPG polyclonal antibody (ab73400, Abcam); anti-OCN monoclonal antibody (ab13418, Abcam).

\section{Preparation of HA and EA-HA}

HA and EA-HA were made into gel form to facilitate application into bone defects. HA gel was made by mixing HA with PEG in a ratio of 1:0.25. EA-HA was prepared by mixing HA powder and EA powder at a ratio of 97:3.

\section{Animals}

Thirty healthy male Wistar rats (Rattus norvegicus) weighing 200 to $250 \mathrm{~g}$ each were obtained from the Biomedic Laboratory in the Faculty of Medicine at the University of Airlangga. The rats were divided into six groups of five animals and were adapted, fed, and provided with water according to standard diet and animal care protocols.

\section{Bone Defect Model}

Before creating the bone defect, all animals were fasted for 12 hours while retaining free access to water. Anesthesia was performed using $100 \mathrm{mg} / \mathrm{kg}$ ketamine hydrochloride (Ketalar, Warner Lambert, Ireland) and $4 \mathrm{mg} / \mathrm{kg}$ xylazine (X1126, Sigma-Aldrich).

A bone defect was created in the lateral femur, $50 \mathrm{~mm}$ from the joint between the tibia and the femur, as a $1 \mathrm{~cm}$ incision with a $0.84 \mathrm{~mm}$ diamond round bur (801G; 018, Mesinger, Germany). This created a defect with dimensions of $2 \mathrm{~mm}$ in diameter and $2 \mathrm{~mm}$ in depth. The bone was irrigated with saline solution during the creation of the bone defect. After drilling, the bone defect groups were treated as described in - Table 1. After treatment, the defects were sutured with nylon (Nylus nylon, nonabsorbable suture, Lotus surgical, India) and topical gentamicin sulfate was applied to the wound at 2 to $4 \mathrm{mg} / \mathrm{kg}$ every 24 hours. Animals were sacrificed after 7 or 14 days of treatment and the femur was dissected for further analysis.

\section{Analysis of Osteoblasts and Osteoclasts}

Histological assessment was performed by hematoxylin-eosin staining and counting the number of osteoblasts and osteoclasts under a microscope at $100 \times$ magnification. Counts were performed by a single operator in five fields of view. ${ }^{13}$

\section{Expression of RANKL, OPG, and OCN}

Histological assessment was performed after immunohistochemical staining by counting the number of macrophages expressing RANKL, OPG, or OCN under a microscope at 400× magnification. Counts were performed by a single operator in five fields of view. ${ }^{13}$

\section{Statistical Analysis}

Data were analyzed using the One-Sample Kolmogorov Smirnov Test for data distribution and the Levene's test for data homogeneity. One-way analysis of variance with least significant difference as a post-hoc test was used to assess

Table 1 Animal treatment groups

\begin{tabular}{|l|l|}
\hline Group & Treatment \\
\hline PEG (control) & The bone defect was treated with PEG. \\
\hline HA & The bone defect was treated with HA gel. \\
\hline EA-HA & The bone defect was treated with EA-HA. \\
\hline
\end{tabular}

Abbreviations: EA-HA, ellagic acid-hydroxyapatite; PEG, polyethylene glycol. 
the expression of RANKL, OPG, and OCN between groups. Results were considered significant at $p<0.05$.

\section{Results}

\section{Osteoblast and Osteoclast Number}

Histological visualization of osteoblasts and osteoclasts is shown in - Fig. 1. The number of osteoblasts in the EA-HA group was higher than in the HA group at day $7(p=0.028)$ and higher than the PEG group on day $14(p=0.017$; -Fig. 1G). The number of osteoclasts in the EA-HA group was lower than either the HA group $(p=0.001)$ or the PEG group $(p=0.000)$ at both 7 and 14 days (- Fig. 1G).

\section{RANKL Protein Expression}

Expression of RANKL in the EA-HA group was lower than in either the HA $(p=0.001)$ or PEG $(p=0.000)$ group at both day 7 and day 14 (vs. HA group, $p=0.000$; vs. PEG group, $p=$ 0.005 ; - Fig. 2). RANKL immunohistochemistry for all groups is shown in - Fig. 3.

\section{OPG Protein Expression}

Expression of OPG in the EA-HA group was higher compared with either the HA group ( $p=0.042 ; p=0.018)$ or the PEG group $(p=0.001 ; p=0.000)$ at day 7 and day 14 , respectively (-Fig. 2). OPG immunohistochemistry for all groups is shown in - Fig. 4.

\section{OCN Protein Expression}

OCN expression in the EA-HA group was higher than in either the HA group $(p=0.000 ; p=0.004)$ or the PEG group $(p=0.016 ; p=0.000)$ on day 7 and day 14 , respectively (- Fig. 2). OCN immunohistochemistry for all groups is shown in - Fig. 5.

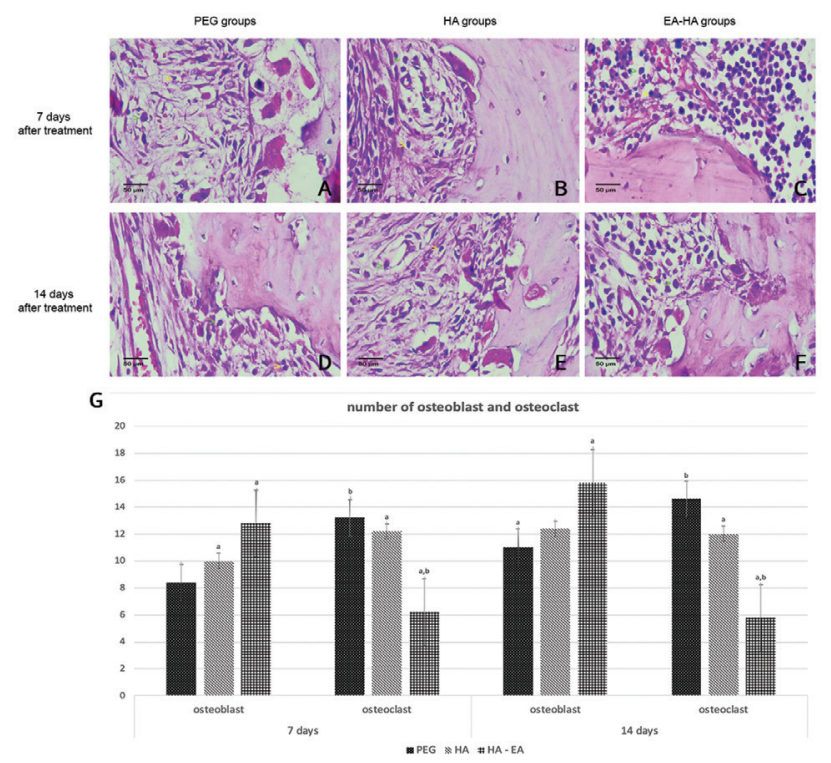

Fig. 1 The numbers of osteoblasts (yellow arrow) and osteoclasts (green arrow) present in all groups $(\mathbf{A}-\mathbf{F})$. The characters above each bar in $(\mathbf{G})$ indicate the significance for each marker $(p<0.05)$.

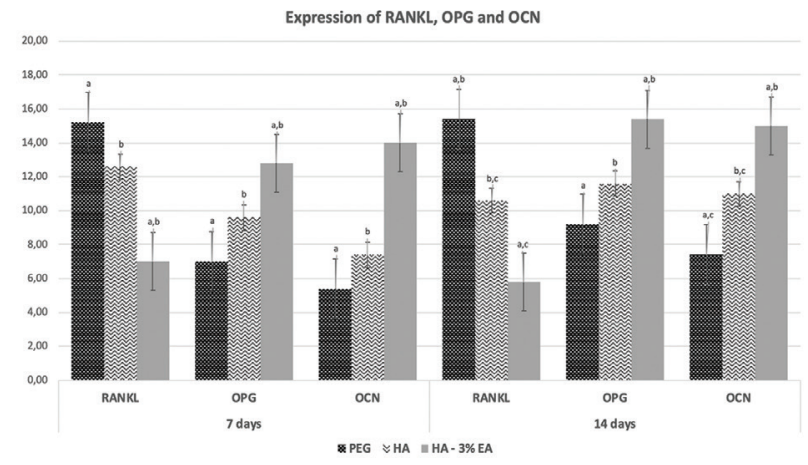

Fig. 2 RANKL, OPG, and OCN expression in all groups. The character above each bar indicates the significance for each marker $(p<0.05)$. OCN, osteocalcin; OPG, osteoprotegerin; RANKL, receptor activator of nuclear factor kappa- $\beta$ ligand.

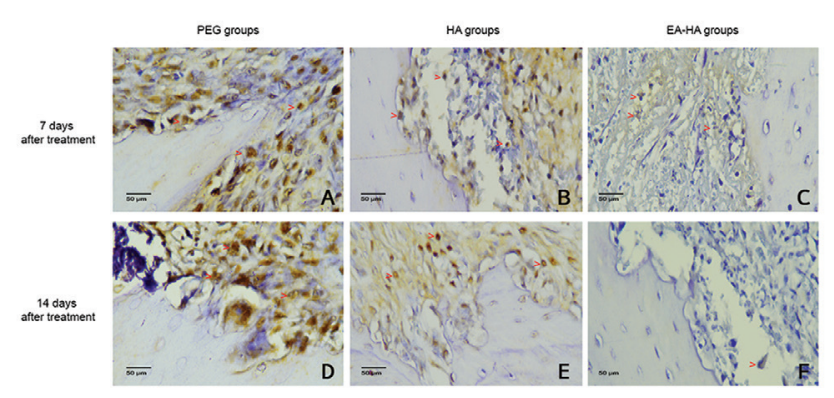

Fig. 3 (A-F) RANKL expression (red arrow) in all groups. Magnification at $\times 400$. RANKL, receptor activator of nuclear factor kappa $-\beta$ ligand.

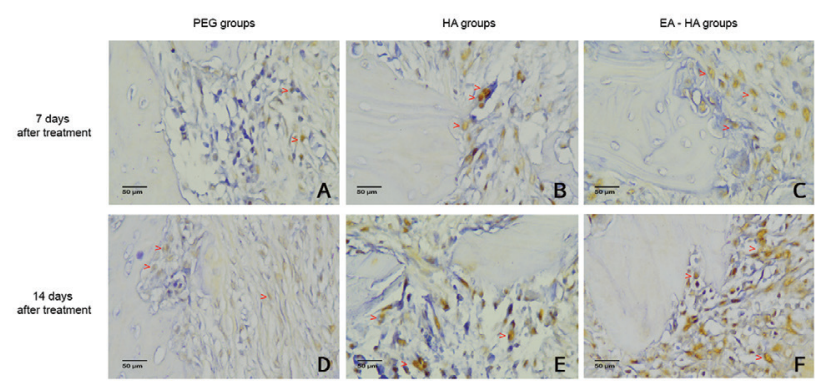

Fig. 4 (A-F) OPG expression (red arrow) in all groups. Magnification at $\times 400$. OPG, osteoprotegerin.

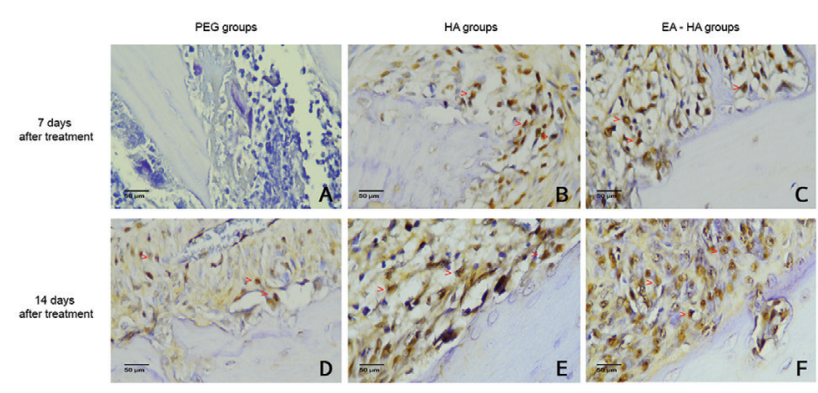

Fig. 5 (A-F) OCN expression (red arrow) in all groups. Magnification at $\times 400$. OCN, osteocalcin. 


\section{Discussion}

Regeneration of a bone defect by osteogenesis begins with inflammation, which is characterized by the formation of blood clots and fibrous tissue. ${ }^{14}$ Macrophages, neutrophils, and polymorphonuclear cells produce cytokines and growth factors such as tumor necrosis factor- $\alpha$, transforming growth factor- $\beta$, fibroblast growth factor, platelet-derived growth factor, and insulin-like growth factor that, alongside collagen, stimulate the migration of mesenchymal cells. After inflammation has resolved, the next stage of regeneration involves the formation of the extracellular matrix and bone. Protein mediators expressed during this phase include bone morphogenetic protein, OCN, and collagen. The collagen forms a matrix around which the deposition and growth of HA crystals occur. The last phase, remodeling, is dominated by OCN, various cytokines, and type I collagen. ${ }^{15-17}$

During the inflammatory phase, oxygen-based free radicals are formed by phagocytic cells such as monocytes, macrophages, and neutrophils located on the bone surface. ${ }^{18}$ These free radicals increase osteoclast formation and bone resorption, which inhibits remineralization of the bone defect or tooth extraction site. ${ }^{19}$ In our study, treatment of the bone defect with HA-EA affected the inflammatory phase of regeneration. This was observed as an inhibition of the bone resorption process; the number of osteoclasts in the EA-HA treatment group was lower than in the control groups. It may be that EA inhibits bone resorption by reducing the production of the pro-inflammatory cytokines that trigger osteoclast differentiation. EA inhibits cytokine production by reducing transcription of NF-kB, resulting in increased osteoclast apoptosis. ${ }^{20}$

The process of osteogenesis is inseparable from the molecular triad of the receptor activator of nuclear factor kappa (RANK), RANKL, and OPG. RANKL has two receptors, RANK and OPG, that are crucial for bone remodeling. RANKL is a regulator of osteoclast formation and activation. In bone, RANKL is expressed by osteoblasts and binds to RANK on osteoclasts. The RANK-RANKL bond accelerates the differentiation of hematopoietic osteoclast precursors into mature osteoclasts. OPG produced by osteoblasts acts as a decoy receptor that competes with RANK to bind RANKL and so inhibits osteoclast differentiation, suppresses osteoclast matrix activation, and induces osteoclast apoptosis. ${ }^{21}$ The more the OPG binds with RANKL, the more the resorption process will be suppressed, and the faster osteogenesis can proceed. ${ }^{22-24}$ Our results show that RANKL expression and osteoclast numbers were decreased by EA-HA while the expression of OPG and OCN and the number of osteoblasts increased. EA has a stimulatory effect on the proliferation of osteoblasts and fibroblasts and is not toxic to bone-forming cells. ${ }^{25}$ It also increases the proliferation of human bone marrow stromal cells (osteoblast progenitor cells) and ${ }^{26}$ inhibits osteoclastogenesis by decreasing the expression of tartrate-resistant acid phosphatase, reducing the amount of RANKL that binds to osteoclasts, and inhibiting resorption by preventing the release of helix peptide chains. ${ }^{27}$ The reduction in RANKL expression by EA-HA effectively increases the expression of OPG. Osteoblasts, which are OPG-secreting cells, are always directly proportional to OPG expression and this condition also confirmed in this research that the number of osteoblasts.

$\mathrm{HA}$ is a potential bone graft material with a composition similar to that of human bone and with good biocompatibility and osteoconductive properties.,28 On application into a bone defect or socket after extraction HA forms a biological apatite layer. This layer produces an environment conducive to recruiting and inducing the proliferation of osteoprogenitor cells and their differentiation into mature osteoblasts. EA has anti-inflammatory, antibacterial, and antioxidant activity and accelerates bone healing by increasing the number of osteoblasts. The EA-HA combination produced the highest number of osteoblasts in the bone defect groups.

EA has polyphenol components that are mostly composed of punicalagin and exhibits antitumor activity. EA concentrations of 1 to $3 \%$ show anti-inflammatory and anticancer activity in lung, prostate, and brain cancer cells. ${ }^{29-31}$ The ellagitannin component of EA is antioxidant, antibacterial, and anti-inflammatory, is nontoxic for normal cells, and accelerates wound healing. ${ }^{16,25}$

OCN is produced by osteoblasts and binds the HA in the bone matrix. ${ }^{32,33}$ It is the main molecular marker in bone formation and bone regeneration. ${ }^{10,34}$ The antioxidant effects of EA reduce oxidative stress in osteoblasts and stimulate their activity, thereby increasing OCN production. The EA-HA treatment group exhibited the highest $\mathrm{OCN}$ expression and the greatest number of osteoblasts. This is consistent with previous reports that EA increases expression of OCN and osteopontin..$^{25,35} \mathrm{EA}$ is also able to inhibit bone resorption by inhibiting the production of cytokines and proteins that trigger osteoclast differentiation. EA inhibits the production of cytokines by inhibiting transcription of NF-kB. This process will increase the differentiation of osteoblasts and osteocytes, increase osteoclast apoptosis, and suppress osteoclast formation, thereby inhibiting bone resorption. ${ }^{20,36}$

\section{Conclusions}

The therapeutic application of HA and EA to treat bone defects increases the number of osteoblasts and expression of OCN and OPG in bone, while reducing the number of osteoclasts and the expression of RANKL.

\section{Conflict of Interest}

None declared.

\section{References}

1 Nainggolan LI, Gunasagaran L. Prevalence of alveolar bone defect pattern in periodontitis patients with diabetes mellitus using bitewing radiography. J Dentomaxillofacial Sci 2018;3(2):88 
2 Susanto H, Nesse W, Kertia N, et al. Prevalence and severity of periodontitis in Indonesian patients with rheumatoid arthritis. J Periodontol 2013;84(8):1067-1074

3 Kuć J, Sierpińska T, Gołębiewska M. Alveolar ridge atrophy related to facial morphology in edentulous patients. Clin Interv Aging 2017;12:1481-1494

4 Levengood SL, Zhang M. Chitosan-based scaffolds for bone tissue engineering. J Mater Chem B Mater Biol Med 2014;2(21):3161-3184

5 Kattimani VS, Kondaka S, Lingamaneni KP. Hydroxyapatitepast, present, and future in bone regeneration. Bone Tissue Regen Insights 2016; 7:BTRI.S36138

6 Polo-Corrales L, Latorre-Esteves M, Ramirez-Vick JE. Scaffold design for bone regeneration. J Nanosci Nanotechnol 2014;14(1):15-56

7 Hashemi ZS, Soleimani M. Tissue engineering scaffolds : history, types and construction methods tissue engineering scaffolds : history, types and construction methods. J Iran Anat Sci 2011;9(35):146-168

8 Medina S, Salazar L, Mejía C, Moreno F. In vitro behavior of the dentin and enamel calcium hydroxyapatite in human premolars subjected to high temperatures. Dyna (Bilbao) 2016;83(195):34-41

9 Dewi AH, Ana ID. The use of hydroxyapatite bone substitute grafting for alveolar ridge preservation, sinus augmentation, and periodontal bone defect: a systematic review. Heliyon 2018;4(10):e00884

10 McKee MD, Pedraza CE, Kaartinen MT. Osteopontin and wound healing in bone. Cells Tissues Organs 2011;194(2-4):313-319

11 Primarizky H, Yuniarti WM, Lukiswanto BS. Ellagic acid activity in healing process of incision wound on male albino rats (Rattus norvegicus) KnE Life Sci 2018;3(6):224

12 Al-Obaidi MM, Al-Bayaty FH, Al Batran R, Hussaini J, Khor GH. Impact of ellagic acid in bone formation after tooth extraction: an experimental study on diabetic rats. Scientific World J 2014;2014:908098

13 Surboyo MDC, Arundina I, Rahayu RP, Mansur D, Bramantoro T. Potential of distilled liquid smoke derived from coconut (Cocos nucifera $\mathrm{L}$ ) shell for traumatic ulcer healing in diabetic rats. Eur J Dent 2019;13(2):271-279

14 Loi F, Córdova LA, Pajarinen J, Lin TH, Yao Z, Goodman SB. Inflammation, fracture and bone repair. Bone 2016;86:119-130

15 Cohen $\mathrm{N}$. Healing processes following tooth extraction in orthodontic cases. J Dentofac Anom Orthod 2014;17:304

16 Shetty S, Kapoor N, Bondu J, Thomas N, Paul T. Bone turnover markers: Emerging tool in the management of osteoporosis. Indian J Endocrinol Metab 2016;20(6):846-52

17 Parra-Torres AY, Valds-Flores M, Orozco L, Velzquez-Cruz R, Molecular Aspects of Bone Remodeling. In: Valds-Flores M, ed. Publicado en: Topics in Osteoporosis. InTech 2013: London, UK. Doi: $10.5772 / 54905$

18 Mountziaris PM, Mikos AG. Modulation of the inflammatory response for enhanced bone tissue regeneration. Tissue Eng Part B Rev 2008;14(2):179-186
19 Agidigbi TS, Kim C. Reactive oxygen species in osteoclast differentiation and possible pharmaceutical targets of ROSmediated osteoclast diseases. Int J Mol Sci 2019;20(14):1-16

20 Ahad A, Ganai AA, Mujeeb M, Siddiqui WA. Ellagic acid, an NF-кB inhibitor, ameliorates renal function in experimental diabetic nephropathy. Chem Biol Interact 2014;219:64-75

21 Tobeiha M, Moghadasian MH, Amin N, Jafarnejad S. RANKL/ RANK/OPG pathway: a mechanism involved in exercise-induced bone remodeling. BioMed Res Int 2020;2020:6910312

22 Wright HL, McCarthy HS, Middleton J, Marshall MJ. RANK, RANKL and osteoprotegerin in bone biology and disease. Curr Rev Musculoskelet Med 2009;2(1):56-64

23 Yamaguchi M. RANK/RANKL/OPG during orthodontic tooth movement. Orthod Craniofac Res 2009;12(2):113-119

24 Belibasakis GN, Bostanci N. The RANKL-OPG system in clinical periodontology. J Clin Periodontol 2012;39(3):239-248

25 Al-Obaidi MM, Al-Bayaty FH, Al Batran R, Hassandarvish P, Rouhollahi E. Protective effect of ellagic acid on healing alveolar bone after tooth extraction in rat-a histological and immunohistochemical study. Arch Oral Biol 2014;59(9):987-999

26 Bickford PC, Tan J, Shytle RD, Sanberg CD, El-Badri N, Sanberg PR. Nutraceuticals synergistically promote proliferation of human stem cells. Stem Cells Dev 2006;15(1):118-123

27 Tanabe S, Santos J, La VD, Howell AB, Grenier D. A-type cranberry proanthocyanidins inhibit the RANKL-dependent differentiation and function of human osteoclasts. Molecules 2011;16(3):2365-2374

28 Maji K, Dasgupta S. Hydroxyapatite-chitosan and gelatin based scaffold for bone tissue engineering. Trans Indian Ceram Soc 2014;73(2):110-114

29 Li J, Wang G, Hou C, Li J, Luo Y, Li B. Punicalagin and ellagic acid from pomegranate peel induce apoptosis and inhibits proliferation in human HepG2 hepatoma cells through targeting mitochondria. Food Agric Immunol 2019;30(1):898-913

30 Weisburg JH, Schuck AG, Reiss SE, et al. Ellagic acid, a dietary polyphenol, selectively cytotoxic to HSC-2 oral carcinoma cells. Anticancer Res 2013;33(5):1829-1836

31 Hussein-Al-Ali SH, Al-Qubaisi M, Rasedee A, Hussein MZ. Evaluation of the cytotoxic effect of ellagic acid nanocomposite in lung cancer A549 cell line and RAW 264.9 cells. J Bionanosci 2017;11(6):578-583

32 Patti A, Gennari L, Merlotti D, Dotta F, Nuti R. Endocrine actions of osteocalcin. Int J Endocrinol 2013;2013:846480

33 Chapurlat RD, Confavreux CB. Novel biological markers of bone: from bone metabolism to bone physiology. Rheumatology (Oxford) 2016;55(10):1714-1725

34 Lovati $A B$, Lopa S, Recordati $C$, et al. In vivo bone formation within engineered hydroxyapatite scaffolds in a sheep model. Calcif Tissue Int 2016;99(2):209-223

35 Paldánius PM, The Role of Osteocalcin in Human Bone Metabolism and Glucose Homeostasis. Vol. 01. Helsinki: University of Helsinki; 2017 1- 95

36 Domazetovic V, Marcucci G, Iantomasi T, Brandi ML, Vincenzini MT. Oxidative stress in bone remodeling: role of antioxidants. Clin Cases Miner Bone Metab 2017;14(2):209-216 